\title{
Combination Regimens of Mirvetuximab Soravtansine, A Folate Receptor Alpha-Targeting Antibody-Drug Conjugate, With Standard-Of-Care Agents Offer Promise for the Treatment of Ovarian Cancer
}

\section{Todd M Bauer*}

Sarah Cannon Research Institute, USA

In a recent issue of Neoplasia, Ponte et al demonstrated the capacity of mirvetuximab soravtansine (IMGN853), a folate receptor alpha (FRa)-targeting antibody-drug conjugate (ADC), to augment the in vitro and in vivo activity of approved chemotherapeutic and biologic agents used in the management of ovarian cancer [1]. These preclinical findings provide a rationale for the further investigation of the antitumor activity of IMGN853 in this malignancy, particularly as part of novel combinatorial strategies with established therapeutic regimens. Current standard-of-care treatment for ovarian cancer, the most lethal gynecologic malignancy, involves debulking surgery typically followed by platinum-based combination chemotherapy [2]. This strategy has changed little in more than a decade and the only marginal improvements seen in terms of survival for this disease over this time underscore the urgent need for new agents and approaches to improve outcomes. An increased understanding of the biological complexity of ovarian tumors has prompted the exploration of targeted strategies designed to shift treatment away from broad-based cytotoxic use towards more tailored interventions [3].

One validated modality involves combining targeted agents, which possess distinct mechanisms of action and favorable tolerability, with established chemotherapeutics-as exemplified by the approval of bevacizumab for use alongside conventional cytotoxic drugs in platinum-resistant, recurrent epithelial ovarian cancer (EOC) [4]. $\mathrm{FRa}$ is a cell surface transmembrane glycoprotein that facilitates the unidirectional transport of folates into cells. In normal tissues, receptor expression is restricted to a variety of polarized epithelia however, aberrant high FRa expression is characteristic of a number of solid tumors, including EOC [5]. This differential distribution pattern has made FRa an attractive candidate for the development of novel, targeted therapeutics. Early approaches to targeting the folate receptor included evaluation of a humanized anti-FRa monoclonal antibody, farletuzumab, to determine whether it would exert clinically relevant antitumor activity through antibody-dependent cell-mediated cytotoxicity and complement-dependent cytotoxicity [6].

In addition, two small molecule folate-cytotoxic agent conjugates, BMS-748285 and vintafolide, have been examined as a means of selectively delivering cytotoxic compounds directly to cancer cells with high folate receptor expression [6]. Although well tolerated in human studies, the clinical development of these agents was limited by a lack of meaningful efficacy in pivotal trials, either as single agents or as part of combination regimens $[7,8]$. The differential expression of FRa and its ability to internalize large molecules makes this receptor well suited for ADC-based strategies. ADCs are complex engineered molecules that comprise a monoclonal antibody, directed towards tumor-associated antigens, conjugated via a stable linker to a potent cytotoxic agent. In this manner, ADCs couple the targeting and pharmacokinetic features of the antibody moiety with the additional cancer-killing impact of the cytotoxic payload [9].

The ADC used in the study, mirvetuximab soravtansine (IMGN853), consists of a humanized FRa-binding monoclonal antibody conjugated to the maytansinoid DM4 [10]. IMGN853 binds with high affinity and specificity to FRa on the surface of tumor cells which, upon antigen binding, promotes ADC internalization and intracellular release of DM4. DM4 subsequently acts as an anti-mitotic agent to inhibit tubulin polymerization and disrupt microtubule assembly, resulting in cell cycle arrest and apoptosis [11]. In early clinical testing, IMGN853 has shown a manageable safety profile and encouraging signs of singleagent activity, particularly in patients with advanced EOC [12]. In an attempt to inform the continued development of IMGN853 as a targeted therapeutic, the authors assessed the combinatorial activity of IMGN853 with a variety of clinically relevant agents in preclinical models of ovarian cancer. They showed that co-treatment of IMGN853 with conventional therapies used in both the front-line (carboplatin) and recurrent settings (pegylated liposomal doxorubicin; PLD) resulted in synergistic anti-proliferative effects in vitro and significantly improved antitumor activity in vivo, including superior efficacy in patient-derived xenograft tumors. Moreover, a noteworthy finding of the study was the markedly improved activity afforded by the combination of IMGN853 and bevacizumab, which caused significant tumor regressions and complete responses in mice bearing platinum-resistant EOC tumors. The mechanism by which IMGN853 achieved improved therapeutic indices in combination with bevacizumab is still under investigation, however it is important to note that in the pivotal studies that led to the approval of bevacizumab for ovarian cancer treatment, efficacy was demonstrated only with regard to progression-free survival, with less reliable effects seen on overall survival-a pattern repeated in more recent clinical evaluations of other anti-angiogenic agents [13].

Thus, it appears that an opportunity exists to improve the efficacy of antiangiogenic therapy in this disease, and it is reasonable to suggest that tailored treatment strategies with optimized combinatorial partners (such as IMGN853) are an area of considerable translational interest. Overall, the demonstrations of combinatorial benefit conferred by the addition of the first FRa-targeting ADC to established therapies provides a compelling framework for the potential application of IMGN853 in the future management of ovarian cancer. In this regard, the ongoing phase Ib trial study in FRa-positive, advanced EOC patients (FORWARD II; NCT02606305) evaluating the safety and efficacy of the ADC alongside bevacizumab, carboplatin, PLD, or pembrolizumab is

*Corresponding author: Todd M Bauer, Sarah Cannon Research Institute/ Tennessee Oncology PLLC, 250 25th Ave N, Nashville TN, 37025, USA, Tel: 615329-7274; Fax: 615524-4939; E-mail: tbauer@tnonc.com

Received February 20, 2017; Accepted April 21, 2017; Published April 28, 2017

Citation: Bauer TM (2017) Combination Regimens of Mirvetuximab Soravtansine A Folate Receptor Alpha-Targeting Antibody-Drug Conjugate, With Standard-OfCare Agents Offer Promise for the Treatment of Ovarian Cancer. Health Care Current Reviews 5: 195. doi: 10.4172/2375-4273.1000195

Copyright: $\odot 2017$ Bauer TM. This is an open-access article distributed under the terms of the Creative Commons Attribution License, which permits unrestricted use, distribution, and reproduction in any medium, provided the original author and source are credited. 
Citation: Bauer TM (2017) Combination Regimens of Mirvetuximab Soravtansine, A Folate Receptor Alpha-Targeting Antibody-Drug Conjugate, With Standard-Of-Care Agents Offer Promise for the Treatment of Ovarian Cancer. Health Care Current Reviews 5: 195. doi: 10.4172/23754273.1000195

warranted. The results of that trial will be informative in determining the feasibility of integrating IMGN853 into novel ovarian cancer treatments and for realizing the full potential of FRa-targeting as a strategy for improving outcomes for patients with this disease.

\section{References}

1. Ponte JF, Ab O, Lanieri L, Lee J, Coccia J, et al. (2016) Mirvetuximab soravtansine (IMGN853), a folate receptor alpha-targeting antibody-drug conjugate, potentiates the activity of standard of care therapeutics in ovarian cancer models. Neoplasia 18: 775-784.

2. Raja FA, Chopra N, Ledermann JA (2012) Optimal first-line treatment in ovarian cancer. Ann Oncol 23: Suppl 10: x118-127.

3. Ledermann JA and Kristeleit RS (2010) Optimal treatment for relapsing ovarian cancer. Ann Oncol 21: Suppl 7: vii218-222.

4. Colombo N, Conte PF, Pignata S, Raspagliesi F, Scambia G (2015) Bevacizumab in ovarian cancer: Focus on clinical data and future perspectives. Crit Rev Oncol Hematol 97: 335- 348.

5. Kalli KR, Oberg AL, Keeney GL, Christianson TJ, Low PS, et al. (2008) Folate receptor alpha as a tumor target in epithelial ovarian cancer. Gynecol Oncol 108: $619-626$

6. Ledermann JA, Canevari S, Thigpen T (2015) Targeting the folate receptor: Diagnostic and therapeutic approaches to personalize cancer treatments. Ann Oncol 26: 2034-2043.
7. Vergote I, Armstrong D, Scambia G, Teneriello M, Sehouli J, et al. (2016) A randomized, double-blind, placebo-controlled, phase iii study to assess efficacy and safety of weekly farletuzumab in combination with carboplatin and taxane in patients with ovarian cancer in first platinum-sensitive relapse. $\mathrm{J}$ Clin Oncol 34: 2271-2278.

8. Merck and Endocyte announce independent DSMB recommends Vintafolide PROCEED Phase 3 trial be stopped for futility following interim analysis.

9. Chari RV, Miller ML, Widdison WC (2014) Antibody-drug conjugates: An emerging concept in cancer therapy. Angew Chem Int Ed Engl 53: 3796-3827.

10. Ab O, Whiteman KR, Bartle LM, Sun X, Singh R, et al. (2015) IMGN853, a folate receptor- $\alpha$ (FRa)-targeting antibody-drug conjugate, exhibits potent targeted antitumor activity against FRa-expressing tumors. Mol Cancer Ther 14: $1605-1613$.

11. Oroudjev E, Lopus M, Wilson L, Audette C, Provenzano C, et al. (2010) Maytansinoid-antibody conjugates induce mitotic arrest by suppressing microtubule dynamic instability. Mole Cancer Ther 9: 2700-2713.

12. Moore KN, Martin LP, O'Malley DM, Matulonis UA, Konner JA, et al. (2017) Safety and activity of mirvetuximab soravtansine (IMGN853), a folate receptor alpha-targeting antibody-drug conjugate, in platinum-resistant ovarian, fallopian tube or primary peritoneal cancer: A phase I expansion study. J Clin Oncol 35: 112-1118.

13. Mahner S, Woelber L, Mueller V, Witzel I, Prieske K, et al. (2015) Beyond Bevacizumab: An outlook to new anti-angiogenics for the treatment of ovarian cancer. Front Oncol 5: 211. 